\title{
Statins for Patients With Non-Alcoholic Fatty Liver Disease
}

\author{
Talal Shakas AlGhamdi
}

\begin{abstract}
Non-alcoholic fatty liver disease (NAFLD) commonly causes an increase in the transaminase levels with the underlying problems, including diabetes, obesity, or both. NAFLD patients are often at an increased risk of cardiovascular events globally, representing a leading cause of death. Therefore, this study aims to review present evidence regarding the utilization of statin in NAFLD patients and discuss the effects of these agents on liver histology and cardiovascular disease. We comprehensively reviewed the current evidence on the statins' safety in NAFLD patients and their effects on cardiovascular events or liver histology. The findings suggest that statins are safe to be administered to NAFLD patients including people with increased transaminase $(<3$ times - upper limit of normal). The reviewed studies indicate that statins may reduce cardiovascular risk. Some controversial data also emerged from the literature regarding the effect of statins on liver histology in NAFLD patients. The treatment with statins is safe, which may also lessen cardiovascular events in patients with NAFLD. Future and ongoing studies will elucidate if statins have a role in treating NAFLD. Although clinicians are often refrained from prescribing statins for NAFLD patients, it is used to reduce cardiovascular mortality and morbidity and lower liver enzymes.
\end{abstract}

Keywords: Hydroxymethylglutaryl-CoA reductase inhibitors; Nonalcoholic steatohepatitis; Atorvastatin; Statins

\section{Introduction}

Non-alcoholic fatty liver disease (NAFLD) is one of the most common and progressive disease of the liver, and it is also considered the leading cause of high serum liver enzyme activities across the developed world [1]. The medical and economic NAFLD burden is because of progressive liver disease and an increased risk of chronic kidney disease, cardiovascular disease (CVD), and some kinds of extrahepatic cancer, such as colorectal cancer or others. Dyslipidemia, diabetes mellitus (DM),

Manuscript submitted October 10, 2020, accepted October 20, 2020

Published online November 29, 2020

Department of Family Medicine, College of Medicine, Majmaah University, P.O. Box 66, Majmaah 11322, Saudi Arabia. Email: ta.alghamdi@mu.edu.sa

doi: https://doi.org/10.14740/jem705 and obesity are some of the most common mechanisms of metabolic syndrome (MetS), which are mostly related to NAFLD; around $75-100 \%$ of the individuals with DM or MetS suffer from NAFLD [2]. NAFLD is most prominently caused by hepatic triglyceride infiltration in the absence of alcohol abuse or chronic liver disease [1]. NAFLD comprises of a spectrum of situations that vary from steatosis to non-alcoholic steatohepatitis (NASH), cirrhosis, fibrosis, or necrosis, which considerably advances to hepatocellular carcinoma [3]. NASH and NAFLD are usually considered hepatic indicators of MetS that are often related to the increased CVD risk [3]. Most of the patients with NAFLD often die due to CVD instead of liver diseases [3,4]. There are no collectively putative treatments for NAFLD [1-4].

Patients with hyperlipidemia, hypertension, diabetes, central obesity, or MetS are usually at an increased risk for developing NAFLD. Such situations tend to have a higher prevalence rate, and NAFLD is now considered a common liver disease cause worldwide, mainly in the USA [5]. In industrialized western countries, around $70-90 \%$ of diabetic patients and $30 \%$ of the general population develop NAFLD [1]. However, the majority of these individuals are asymptomatic, and the liver enzymes are either found to be normal or elevated. To be diagnosed with NAFLD, it is crucial to dismiss alcoholic hepatitis with hereditary hemochromatosis, medical history, Wilson's disease, viral hepatitis, and autoimmune hepatitis with laboratory testing. The results from an ultrasound often show an accumulation of fats in the liver. Some patients may complain regarding the pain in the right upper quadrant, which is usually not associated with NAFLD. An enlarged liver is sometimes found in a few patients during physical examination.

NAFLD-related morbidity, together with NASH, will dramatically elevate in the coming years, prominently in the Gulf countries, where there is already an epidemic of DM [6] or obesity [7]. The mortality of NAFLD is higher in comparison to the general population, primarily due to $\operatorname{CVD}[8,9]$. An increase in weight loss and physical activity is the sole therapy with a definite indication for its advantage [10]. At the same time, other treatments, for example, insulin-sensitizing drugs (pioglitazone or metformin), might be useful [11]. Unexpectedly, atorvastatin is thought to reduce aminotransferase levels in NAFLD patients [12, 13]; however, clinicians most often get concerned with respect to statin prescription in patients with increased liver enzymes. A study conducted in the USA from 937 primary care physicians revealed that $50 \%$ of physicians in primary care might not recommend statins for patients whose liver enzymes are $1.5 \times$ the upper limit of normal (ULN) [14].

Contemporary reports suggest that "3-hydroxy-3-me- 
thyl-glutaryl-coenzyme A (HMG-CoA) reductase inhibitors" (statins) tends to normalize enzymes in the liver as well as increase liver aminotransferases levels in individuals with NAFLD [15]. Either this a steady effect or if it leads to improvements in the clinical outcomes beyond normalization of atypical liver enzymes is typically not clear. Therefore, this review aims to review the most current evidence concerning the use of statin in NAFLD patients and discuss the effects of these agents on liver histology and CVD. To the best of our knowledge, no such review has been conducted in the review has addressed such inquiries in NAFLD individuals.

\section{Methodology}

A comprehensive review of previous evidence was conducted to categorise research articles evaluating the statin use and its effects in NAFLD patients. The search terms included "nonalcoholic fatty liver disease", "hydroxymethylglutaryl-CoA reductase inhibitors", "non-alcoholic steatohepatitis", "HMGCoA reductase inhibitors", "atorvastatin", "statins", "mevastatin", "lovastatin", "fluvastatin", "cerivastatin", "atorvastatin", "simvastatin", "rosuvastatin", "pravastatin", "pitavastatin", "safety", and "therapeutic use". The research articles conducted in the English language were screened for inclusion. We also emphasized upon the data published after the latest NAFLD guidelines [16]. Studies performed on animals were also excluded. To include additional references, the bibliographies of the selected articles were also included and reviewed.

\section{NAFLD Prevalence}

Around $30 \%$ of the people aged $\geq 15$ years of age in 2015 suffered from NAFLD. After adjusting for a lower prevalence in the population aged $<15$ years, the prevalence in all age groups was found to be $24.8 \%$ in Saudi Arabia [17]. Regarding the distribution of gender and age of NAFLD population, data from the general population studies in several other countries were applied, where the prevalence was 1.3 times higher in males than females with an increased prevalence with age [18, 19]. Younger age groups were not included in the prevalence studies and it was expected that the rates of prevalence might decrease with declining age. In Saudi Arabia, a study with NAFLD prevalence employed computed tomography (CT) scan data from 100 adult hospital patients in 2012, where it was reported that $18-54 \%$ NAFLD mainly depends on the applied criteria [20]. Another study conducted on 230 patients with DM at Jazan General Hospital in 2013 reported a 47.8\% of the prevalence. From these patients, there was a slightly high prevalence in males $(49.1 \%)$ than females $(46.3 \%)$, whereas this prevalence was $52.9 \%$ among the population of middleaged individuals (ages 40 - 59 years) [21].

\section{Statins Safety in NAFLD Patients}

Many observational studies propose that statins are benign in people with increased levels of transaminase. The patient did not indicate alcohol abuse or hepatitis $\mathrm{C}$ or $\mathrm{B}$ in these patients; hence, NAFLD was the most possible cause of increased transaminase [22]. Statin-induced hepatotoxicity is often related to an asymptomatic increase of serum aminotransferases within 12 weeks of therapy, with no histopathological fluctuations found in the liver [23]. Many phases II and III clinical trials have shown an increase in alanine aminotransferase (ALT) or aspartate aminotransferase (AST).

Onofrei [24] demonstrated many studies to provide evidence that usage of statins is related to increased transaminases. One of the retrospective investigations shown that of the 2,587 patients with increase liver function tests (LFTs) at baseline, there were around 342 patients that were exposed to therapy with statins. ALT/AST increase has also been demonstrated to be reliant on dose [8]. Most of the dosages of rosuvastatin, atorvastatin, simvastatin, pravastatin, and lovastatin are accompanied by modest but noteworthy rise in the levels of transaminase [25]. Such an increase creates issues that statins can aggravate the disease of the liver. This issue also resulted in drug manufacturers that recommended that statin therapy shall be avoided in individuals having unexplained aminotransferase increase and underlying liver disease. In the past, it was, however, suggested that individuals who consumed statin medications need to have their LFTs checked at baseline, after 12 weeks of dose amplification [24]. This spontaneous rise in the levels of aminotransferase leads to a transient increase that attributes to the reduction of cholesterol with alcohol consumption, obesity, DM, statin treatment, or other medications [7]. Multiple studies have been performed to check if statin therapy contributes to or causes significant damage to the liver. One of the "Adverse Event Database Merck Worldwide" studied 232 acute liver failure cases, which was related to lovastatin. The study found a risk for fulminant liver failure to be two per one million individuals [23].

From 1990 to 2002, around 51,741 liver transplantations in the USA were undertaken. Of these transplantations, most prominently, only three surgeries were associated with statin therapy [24]. A retrospective cohort study was executed by Avins [26] to examine the hepatic effects of exposure with lovastatin in the individuals having liver disease. The primary outcomes were to examine if there was any form of livertest abnormalities related to poor prognosis among individuals with drug-induced liver disease with respect to Hy's law. Likewise, Avins et al [26] suggested that Hy's law is defined as a "profile of liver test that is predominantly related to poor prognosis for drug-induced liver disease patients" [26]. Other secondary outcomes comprise of injury of the liver as well as liver failure or clinical cirrhosis development. Exposure of Lovastatin is often associated with a reduced incidence of severe to moderate liver injury, along with the occurrence of either liver failure or cirrhosis. It was therefore concluded in this study that lovastatin is not related to an increased risk of adverse hepatic outcomes. Around 1,081 patients at baseline, had an increased transaminase levels ( $<2$ times the ULN), while the treatment with either atorvastatin $80 \mathrm{mg}$ /day or simvastatin 20 - $40 \mathrm{mg} /$ day reduced the levels of transaminase [27]. 


\section{Statin Effect on the Cardiovascular Morbidity in NAFLD Patients}

At present, post-hoc examination of three randomized controlled trials (RCTs) indicated that cardiovascular events may decline through statins in individual with increases levels of transaminase, seemingly, because of NAFLD. One study used the post-hoc Greek Atorvastatin and Coronary Heart Disease Evaluation (GREACE) trial to perform treatment with atorvastatin for 3 years, limiting cardiovascular events by $39 \%$ in an individual with normal transaminase levels [28]. On the contrary, atorvastatin limited the cardiovascular events by $68 \%$ in coronary heart disease (CHD) individuals along with an increase in the levels of transaminase $(\mathrm{P}=0.0074 \mathrm{com}$ pared to the decrease in CVD risk in patients having normal transaminase levels) [28]. With respect to the post-hoc examination of the ATTEMPT study, atorvastatin treatment for 42 months aimed at low-density lipoprotein cholesterol (LDL-C) levels $<100 \mathrm{mg} / \mathrm{dL}$ in people with MetS and increased levels of transaminase that limited the cardiovascular events more in comparison to the atorvastatin treatment targeting at LDL-C (P $=0.024)$ levels $<130 \mathrm{mg} / \mathrm{dL}$ [29]. Lastly, another post-hoc investigation of the IDEAL trial, atorvastatin $(80 \mathrm{mg} /$ day $)$ treatment often lessen the cardiovascular events more as compared to the simvastatin $(20-40 \mathrm{mg} /$ day $)$ solely in CHD patients and increased levels of transaminase and, not in those with normal levels of transaminase $(\mathrm{P}$ for heterogeneity $=0.0277)$ [27]. Therefore, the data obtained on a preliminary basis indicates that statins often reduced the cardiovascular morbidity in NAFLD patients. Simultaneously, the NAFLD presence does not differentiate the dyslipidemia management based on both the US and European guidelines [30, 31].

\section{Statins Effects on Liver Histology in NAFLD Individuals}

Given the involvement of the oxidative stress, increase in apoptosis in the NAFLD pathogenesis and subclinical inflammation, statins are responsible for improving the liver histology in such patients using the pleiotropic effects [32]. On the contrary, few small uncontrolled studies utilized CT or ultrasound to examine the effects of the statins on liver steatosis in humans. The majority of these studies were known to show improvement [33, 34]. In one of a larger RCT, atorvastatin and combined treatment (atorvastatin + fenofibrate) were more effective than fenofibrate alone, while they also reduced the liver echogenicity [35]. Another RCT indicated that atorvastatin $10-20 \mathrm{mg}$ /day was considered effectual as pitavastatin $2-4$ $\mathrm{mg}$ /day in dropping the hepatic steatosis in CT [36]. However, very limited data was available with respect to the statins' effects on liver histology. Many studies with small sample size and uncontrolled prospective design examined the effects of the statins on the histology of the liver in NAFLD individuals. The majority of such studies confirmed that treatment with statins often reduced hepatic steatosis [37,38], while in others, no change was reported [39]. In some of the research studies, improvement in inflammation was found [38], while in others, this was not reported. Fibrosis was not found to be reduced in any other study except in a small introductory report in six patients who were treated with rosuvastatin for 6 months [38].

\section{Conclusions}

There has been a remarkable advancement in the knowledge of the natural history, prevalence, and pathogenesis of the NAFLD. However, at present, no actual novelties concerning therapeutic management exist. Statins are considered safe in individuals with pre-eminent transaminase levels because of NAFLD. A post-hoc analysis of the RCT's indicates that statins may decrease CVD morbidity in the studied population. On the contrary, the effects of the statins on liver histology in NAFLD individuals are still controversial. Future and ongoing RCTs will help to clarify if statins have a role to play in NAFLD treatment. More research is needed to detect NAFLD and on the best way to detect the presence of advanced fibrosis.

\section{Acknowledgments}

None to declare.

\section{Financial Disclosure}

This research did not receive any specific grant from funding agencies in the public, commercial, or not-for-profit sectors.

\section{Conflict of Interest}

None to declare.

\section{Author Contributions}

The author wrote and read the manuscript.

\section{Data Availability}

The authors declare that data supporting the findings of this study are available within the article.

\section{References}

1. Angulo P. Nonalcoholic fatty liver disease. N Engl J Med. 2002;346(16):1221-1231.

2. Eguchi A, Povero D, Alkhouri N, Feldstein AE. Novel therapeutic targets for nonalcoholic fatty liver disease. Expert Opin Ther Targets. 2013;17(7):773-779.

3. Athyros VG, Tziomalos K, Daskalopoulos GN, Karagian- 
nis A, Mikhailidis DP. Statin-based treatment for cardiovascular risk and non-alcoholic fatty liver disease. Killing two birds with one stone? Ann Med. 2011;43(3):167-171.

4. Bieghs V, Rensen PC, Hofker MH, Shiri-Sverdlov R. NASH and atherosclerosis are two aspects of a shared disease: central role for macrophages. Atherosclerosis. 2012;220(2):287-293.

5. Kim $\mathrm{CH}$, Younossi ZM. Nonalcoholic fatty liver disease: a manifestation of the metabolic syndrome. Cleve Clin J Med. 2008;75(10):721-728.

6. Abuyassin B, Laher I. Diabetes epidemic sweeping the Arab world. World J Diabetes. 2016;7(8):165-174.

7. DeNicola E, Aburizaiza OS, Siddique A, Khwaja H, Carpenter DO. Obesity and public health in the Kingdom of Saudi Arabia. Rev Environ Health. 2015;30(3):191-205.

8. Soderberg C, Stal P, Askling J, Glaumann H, Lindberg G, Marmur J, Hultcrantz R. Decreased survival of subjects with elevated liver function tests during a 28 -year followup. Hepatology. 2010;51(2):595-602.

9. Targher G, Day CP, Bonora E. Risk of cardiovascular disease in patients with nonalcoholic fatty liver disease. N Engl J Med. 2010;363(14):1341-1350.

10. Promrat K, Kleiner DE, Niemeier HM, Jackvony E, Kearns M, Wands JR, Fava JL, et al. Randomized controlled trial testing the effects of weight loss on nonalcoholic steatohepatitis. Hepatology. 2010;51(1):121-129.

11. Angelico F, Burattin M, Alessandri C, Del Ben M, Lirussi F. Drugs improving insulin resistance for non-alcoholic fatty liver disease and/or non-alcoholic steatohepatitis. Cochrane Database Syst Rev. 2007;(1):CD005166.

12. Hyogo H, Tazuma S, Arihiro K, Iwamoto K, Nabeshima $\mathrm{Y}$, Inoue M, Ishitobi T, et al. Efficacy of atorvastatin for the treatment of nonalcoholic steatohepatitis with dyslipidemia. Metabolism. 2008;57(12):1711-1718.

13. Georgescu EF, Georgescu M. Therapeutic options in nonalcoholic steatohepatitis (NASH). Are all agents alike? Results of a preliminary study. J Gastrointestin Liver Dis. 2007;16(1):39-46.

14. Rzouq FS, Volk ML, Hatoum HH, Talluri SK, Mummadi RR, Sood GK. Hepatotoxicity fears contribute to underutilization of statin medications by primary care physicians. Am J Med Sci. 2010;340(2):89-93.

15. Eslami L, Merat S, Malekzadeh R, Nasseri-Moghaddam $\mathrm{S}$, Aramin H. Statins for non-alcoholic fatty liver disease and non-alcoholic steatohepatitis. Cochrane Database Syst Rev. 2013;12:CD008623.

16. Chalasani N, Younossi Z, Lavine JE, Diehl AM, Brunt EM, Cusi K, Charlton M, et al. The diagnosis and management of non-alcoholic fatty liver disease: practice Guideline by the American Association for the Study of Liver Diseases, American College of Gastroenterology, and the American Gastroenterological Association. Hepatology. 2012;55(6):2005-2023.

17. Alswat K, Aljumah AA, Sanai FM, Abaalkhail F, A1ghamdi M, Al Hamoudi WK, Al Khathlan A, et al. Nonalcoholic fatty liver disease burden - Saudi Arabia and United Arab Emirates, 2017-2030. Saudi J Gastroenterol. 2018;24(4):211-219.

18. Lazo M, Hernaez R, Eberhardt MS, Bonekamp S, Kamel
I, Guallar E, Koteish A, et al. Prevalence of nonalcoholic fatty liver disease in the United States: the Third National Health and Nutrition Examination Survey, 1988-1994. Am J Epidemiol. 2013;178(1):38-45.

19. Caballeria L, Pera G, Auladell MA, Toran P, Munoz L, Miranda D, Aluma A, et al. Prevalence and factors associated with the presence of nonalcoholic fatty liver disease in an adult population in Spain. Eur J Gastroenterol Hepatol. 2010;22(1):24-32.

20. Alshumrani GA, Shawky KA, Assiri YI, Algathradi MA, Mahfouz AA, Mostafa OA. Fatty liver disease among adults in Southwestern Saudi Arabia. Med J Cairo Univ 2013; 81:205-209.

21. Elmakki E, Aqeely H, Bani I, Omer H, Solan Y, Taher A, et al. Nonalcoholic Fatty Liver Disease (NAFLD) in Saudi patients with T2DM in Jazan region: prevalence and associated factors. BJMMR 2015; 5:872-879.

22. Younossi ZM, Stepanova M, Afendy M, Fang Y, Younossi Y, Mir H, Srishord M. Changes in the prevalence of the most common causes of chronic liver diseases in the United States from 1988 to 2008. Clin Gastroenterol Hepatol. 2011;9(6):524-530 e521; quiz e560.

23. Tzefos M, Olin JL. 3-hydroxyl-3-methylglutaryl coenzyme A reductase inhibitor use in chronic liver disease: a therapeutic controversy. J Clin Lipidol. 2011;5(6):450459.

24. Onofrei MD, Butler KL, Fuke DC, Miller HB. Safety of statin therapy in patients with preexisting liver disease. Pharmacotherapy. 2008;28(4):522-529.

25. Gillett RC, Jr., Norrell A. Considerations for safe use of statins: liver enzyme abnormalities and muscle toxicity. Am Fam Physician. 2011;83(6):711-716.

26. Avins AL, Manos MM, Ackerson L, Zhao W, Murphy R, Levin TR, Watson DJ, et al. Hepatic effects of lovastatin exposure in patients with liver disease: a retrospective cohort study. Drug Saf. 2008;31(4):325-334.

27. Tikkanen MJ, Fayyad R, Faergeman O, Olsson AG, Wun CC, Laskey R, Kastelein JJ, et al. Effect of intensive lipid lowering with atorvastatin on cardiovascular outcomes in coronary heart disease patients with mild-to-moderate baseline elevations in alanine aminotransferase levels. Int J Cardiol. 2013;168(4):3846-3852.

28. Athyros VG, Tziomalos K, Gossios TD, Griva T, Anagnostis P, Kargiotis K, Pagourelias ED, et al. Safety and efficacy of long-term statin treatment for cardiovascular events in patients with coronary heart disease and abnormal liver tests in the Greek Atorvastatin and Coronary Heart Disease Evaluation (GREACE) Study: a post-hoc analysis. Lancet. 2010;376(9756):1916-1922.

29. Athyros VG, Giouleme O, Ganotakis ES, Elisaf M, Tziomalos K, Vassiliadis T, Liberopoulos EN, et al. Safety and impact on cardiovascular events of long-term multifactorial treatment in patients with metabolic syndrome and abnormal liver function tests: a post hoc analysis of the randomised ATTEMPT study. Arch Med Sci. 2011;7(5):796-805.

30. European Association for Cardiovascular Prevention, Rehabilitation, Reiner Z, Catapano AL, De Backer G, Graham I, Taskinen MR, et al. ESC/EAS Guidelines for the 
management of dyslipidaemias: the Task Force for the management of dyslipidaemias of the European Society of Cardiology (ESC) and the European Atherosclerosis Society (EAS). Eur Heart J. 2011;32(14):1769-1818.

31. Stone NJ, Robinson JG, Lichtenstein AH, Bairey Merz CN, Blum CB, Eckel RH, Goldberg AC, et al. 2013 ACC/ AHA guideline on the treatment of blood cholesterol to reduce atherosclerotic cardiovascular risk in adults: a report of the American College of Cardiology/American Heart Association Task Force on Practice Guidelines. Circulation. 2014;129(25 Suppl 2):S1-45.

32. Tziomalos K. Lipid-lowering agents in the management of nonalcoholic fatty liver disease. World J Hepatol. 2014;6(10):738-744.

33. Hatzitolios A, Savopoulos C, Lazaraki G, Sidiropoulos I, Haritanti P, Lefkopoulos A, Karagiannopoulou G, et al. Efficacy of omega-3 fatty acids, atorvastatin and orlistat in non-alcoholic fatty liver disease with dyslipidemia. Indian J Gastroenterol. 2004;23(4):131-134.

34. Kimura Y, Hyogo H, Yamagishi S, Takeuchi M, Ishitobi T, Nabeshima Y, Arihiro K, et al. Atorvastatin decreases serum levels of advanced glycation endproducts (AGEs) in nonalcoholic steatohepatitis (NASH) patients with dyslipidemia: clinical usefulness of AGEs as a biomarker for the attenuation of NASH. J Gastroenterol. 2010;45(7):750-757.

35. Athyros VG, Mikhailidis DP, Didangelos TP, Giouleme
OI, Liberopoulos EN, Karagiannis A, Kakafika AI, et al. Effect of multifactorial treatment on non-alcoholic fatty liver disease in metabolic syndrome: a randomised study. Curr Med Res Opin. 2006;22(5):873-883.

36. Han KH, Rha SW, Kang HJ, Bae JW, Choi BJ, Choi SY, Gwon HC, et al. Evaluation of short-term safety and efficacy of $\mathrm{HMG}-\mathrm{CoA}$ reductase inhibitors in hypercholesterolemic patients with elevated serum alanine transaminase concentrations: PITCH study (PITavastatin versus atorvastatin to evaluate the effect on patients with hypercholesterolemia and mild to moderate hepatic damage). J Clin Lipidol. 2012;6(4):340-351.

37. Georgescu EF, Georgescu M. Therapeutic options in nonalcoholic steatohepatitis (NASH). Are all agents alike? Results of a preliminary study. J Gastrointestin Liver Dis. 2007;16:39-46.

38. Kargiotis K, Katsiki N, Athyros VG, Giouleme O, Patsiaoura K, Katsiki E, Mikhailidis DP, et al. Effect of rosuvastatin on non-alcoholic steatohepatitis in patients with metabolic syndrome and hypercholesterolaemia: a preliminary report. Curr Vasc Pharmacol. 2014;12(3):505511.

39. Nakahara T, Hyogo H, Kimura Y, Ishitobi T, Arihiro K, Aikata H, Takahashi S, et al. Efficacy of rosuvastatin for the treatment of non-alcoholic steatohepatitis with dyslipidemia: An open-label, pilot study. Hepatol Res. 2012;42(11):1065-1072. 